Research Paper

\title{
Robo 4 - the double-edged sword in prostate cancer: impact on cancer cell aggressiveness and tumor vasculature
}

\author{
Andreas Pircher ${ }^{1 \#, ~ G e o r g ~ S c h a ̈ f e r ~}{ }^{2 \#}$, Andrea Eigentler ${ }^{3}$, Renate Pichler², Martin Puhr ${ }^{3}$, Eberhard Steiner ${ }^{3}$, \\ Wolfgang Horninger ${ }^{3}$, Eberhard Gunsilius ${ }^{1}$, Helmut Klocker ${ }^{3}$ and Isabel Heidegger ${ }^{3 凶}$ \\ 1. Department of Hematology and Oncology, Internal Medicine V, Medical University Innsbruck, Austria \\ 2. Department of Pathology, Medical University Innsbruck, Austria \\ 3. Department of Urology, Medical University Innsbruck, Austria \\ \# both are first authors \\ $\square$ Corresponding author: Isabel Heidegger, MD, PhD, Associate Professor of Urology, Anichstreet 35, 6020 Innsbruck, Austria. \\ Isabel-maria.heidegger@i-med.ac.at; Tel: 0043512504 24808; Fax: 004351250424898 \\ (c) Ivyspring International Publisher. This is an open access article distributed under the terms of the Creative Commons Attribution (CC BY-NC) license \\ (https://creativecommons.org/licenses/by-nc/4.0/). See http://ivyspring.com/terms for full terms and conditions.
}

Received: 2018.07.24; Accepted: 2018.11.09; Published: 2019.01.01

\begin{abstract}
Background: The magic roundabout receptor 4 (Robo 4) is a tumor endothelial marker expressed in the vascular network of various tumor entities. However, the role of Robo 4 in prostate cancer $(\mathrm{PCa})$, the second common cause of cancer death among men in -developed countries, has not been described yet. Thus, the present study investigates for the first time the impact of Robo 4 in PCa both in the clinical setting and in vitro.

Methods and Results: Immunohistochemical analyses of benign and malignant prostate tissue samples of $95 \mathrm{PCa}$ patients, who underwent radical prostatectomy (RPE), revealed a significant elevated expression of Robo 4 as well as its ligand Slit 2 protein in cancerous tissue compared to benign. Moreover, increased Robo 4 expression was associated with higher Gleason score and PT stage. In advanced stage we observed a hypothesis-generating trend that high Robo 4 and Slit 2 expression is associated with delayed development of tumor recurrence compared to patients with low Robo 4 and Slit 2 expression, respectively.

In contrast to so far described exclusive expression of Robo 4 in the tumor vascular network, our analyses showed that in PCa Robo 4 is not only expressed in the tumor stroma but also in cancer epithelial cells. This finding was also confirmed in vitro as PC3 PCa cells express Robo 4 on mRNA as well as protein level. Overexpression of Robo 4 in PC3 as well as in Robo 4 negative DU145 and LNCaP PCa cells was associated with a significant decrease in cell-proliferation and cell-viability.

Conclusion: In summary we observed that Robo 4 plays a considerable role in PCa development as it is expressed in cancer epithelial cells as well as in the surrounding tumor stroma. Moreover, higher histological tumor grade was associated with increased Robo 4 expression; controversially patients with high Robo 4 tend to exert lower biochemical recurrence possibly reflecting a protective role of Robo 4.
\end{abstract}

Key words: Prostate cancer, Robo 4, Slit 2, cancer aggressiveness, tumor recurrence

\section{Introduction}

Prostate cancer (PCa) is the most common malignancy in men and the second common cause of cancer death among men in European countries (Siegel et al., 2016). While organ confined PCa is mostly cured by local therapies like radical prostatectomy (RPE), radiation therapy (plus anti-androgenic therapy in intermediate and high risk cancers) or focal therapy about $30 \%$ of prostate 
tumors are diagnosed in a locally advanced or primary metastatic stage. In addition to androgen receptor (AR) regulation, one of the major steps in $\mathrm{PCa}$ progression, new blood vessel formation (angiogenesis) plays a major role in tumor promotion and metastatic PCa growth $(1,2)$.

Inhibition of angiogenesis is an attractive treatment option. Currently most anti-angiogenic strategies inhibit the vascular endothelial growth factor / receptor (VEGF/R) signaling pathway $(2,3)$. Both treatment strategies (VEGF neutralizing antibodies or VEGFR tyrosine kinase inhibitors) proved clinical efficacy in several tumor entities, however therapy success is hampered by development of evasive resistance or already pre-existing intrinsic refractoriness (4-6). In line with these observations first clinical studies in PCa using anti-angiogenic drugs showed disappointing results, thus calling for a better understanding of molecular mechanisms of angiogenesis in $\mathrm{PCa}$ (7) (www.clinicaltrials.org).

Genome analyses of endothelial cells identified various genes specifically expressed in tumor endothelial cells called tumor endothelial markers (TEMs). In general, the roundabouts are transmembrane receptors expressed in developing tissues, such as the central nervous system (Robo 1, Robo 2, Robo 3) and neovascular endothelium (Robo 4) (8). Robo 4, also referred to as "magic roundabout," is an endothelial specific guidance receptor expressed at sites of active angiogenesis. In particular, Robo 4 is elevated in the tumor vasculature and down-regulated in the mature vasculature, suggesting that Robo 4 may be a useful neo-vessel marker for noninvasive detection and characterization of nascent cancers undergoing active angiogenesis (9). Functionally, Robo 4 signaling induces inhibition of endothelial cell migration and is partially mediated by interference with the Ras-Raf-Mek-Erk pathway (9, 10). The corresponding ligands of Robo 4 are the Slit proteins, which are large secreted proteins encoded by a family of three genes (Slit 1-3). Slit 2 was found to interact with Robo 4, to modulate endothelial cell migration and to participate in tumor angiogenesis (11).

Best to our knowledge, the clinical impact of the TEM Robo 4 has not been investigated in PCa so far. Therefore, we investigated the role of Robo 4 in localized and advanced PCa in both the clinical and preclinical setting.

\section{Patients, Material and Methods}

\section{Patients and data acquisition}

Demographic data of 167 patients with biopsy-verified PCa were included in the study. 95/167 patients underwent an open retropubic or robotic assisted (Da Vinci) radical prostatectomy (RPE) at our department. Additionally, we performed a long-term follow-up analysis of these patients including regular measurement of PSA levels. Use of archived tissue samples for this study was approved by the Ethics Committee of the Medical University Innsbruck (UN3174, AM 3174), informed consent of all patients included in the study is available.

\section{Tissue microarray and immunohistochemistry}

To evaluate differences in Robo 4 expression between malignant and benign prostate tissue, we constructed a tissue microarray (TMA) of 96 patients with PCa who underwent RPE. In addition, punches of fresh frozen paraffin embedded metastatic PCa cell lines (PC3, DU145, PC3-DR, and DU145-DR) were included. For each selected case, three cancer tissue cores and three benign cores were punched. The TMA was assembled using a manual tissue arrayer (Beecher Instruments, Sun Prairie, WI). Hematoxilin/Eosin (HE) and p63/-methylacyl-CoA racemase (AMACR) immunohistochemistry (IHC) double staining to control the histological diagnosis and Robo 4, Slit 2 and CD31 IHC were performed on a Discovery-XT staining device (Ventana, Tucson, AZ) using the following antibodies: anti-Robo 4 (Abcam), anti-Slit 2 (Abcam), anti-CD31 (Dako), anti-p63 (Sigma-Aldrich), anti-AMACR (Dako). Microscope images were taken with a Zeiss Imager Z2 microscope (Zeiss, Vienna) equipped with a Pixelink PLB622-CU camera (Canimpex Enterprises Ltd, Halifax, NS, Canada). IHC expression analysis was performed by an experienced uropathologist (G.S) as well as independently by A.P. by multiplying the percentage of positive cells with the staining intensity ( 0 : no point, weak light: 1 point, medium: 2 points, strong: 3 points). Micro vessel density (MVD) was defined as the number of CD31 positively stained vessels per TMA core (12).

\section{Cell lines and Cell culture}

PC3, DU145, CW22RV1 and LNCaP cell lines were obtained from the American Type Culture Collection (ATCC). DUCaP were obtained from Professor J. Schalken (Center for Molecular Life Science, Nijmegen, Netherlands), LAPC-4 cells were a gift from Professor A. Cato (Karlsruhe Institute of Technology, Karlsruhe, Germany). Human endothelial vein cells (HUVEC) were a kind gift of Professor Dr. R. Kirchmair (Medical University Innsbruck, Austria). The subline LNCaP Abl was established by our group after long term cultivation of LNCaP in steroid free medium (13). LAPC4 cells were 
cultured in the presence of increasing doses of enzalutamide (LAPC-4 EnzaR), abiraterone (LAPC-4 AbiR) or vehicle $(\mathrm{EtOH})$ as described previously by our group $(14,15)$ to generate drug-resistant sublines. Cell lines were cultured in growth media with supplements as previously described (16-19). The identity of the used cancer cell lines was confirmed by forensic DNA fingerprinting methods using the AmpFISTR ${ }^{\circledR}$ SGM Plus ${ }^{\circledR}$ PCR amplification kit (Applied Biosystems).

\section{Overexpression experiments}

150.000 cells (PC3, DU145, LNCaP) per well were seeded into 6 well plates. On the next day cells were transfected with $1 \mu \mathrm{g}$ of the following expression plasmids: Robo 4 (human cDNA clone Robo 4 (NM_019055), Origene, SC113316) or pCMV6 empty vector using X-tremeGENE HP DNA transfection reagent (Roche) following to the suppliers' protocol. 96h after transfection cells were harvested. Target gene overexpression was confirmed by qRT-PCR and Western blot analysis.

\section{Knock down experiments}

150.000 (PC3, DU145, LNCaP) cells per well were seeded into 6 well plates. Transfection of receptor-targeting or control siRNAs was performed the following day using Lipofectamin 2000 transfection reagent (Invitrogen) according to the manufacturer's instruction. $40 \mathrm{nM}$ siCtrl (ON-TARGET plus non-targeting Pool, Dharmacon, D-001810-10) and siRNA Robo 4 (ON-TARGET plus Human Robo 4 siRNA-SMART pool, Dharmacon, L-015216-01) was used. Target gene downregulation was confirmed by qRT-PCR.

\section{Quantitative real-time PCR (qRT-PCR)}

Total RNA was isolated using the RNeasy mini kit (Qiagen). cDNA synthesis was performed using iscript select cDNA synthesis kit (Bio-Rad Laboratories). qRT-PCR was performed on an ABI Prism 7500 fast real-time PCR System (Applied Biosystems, Life Technologies). A TaqMan Assay Hs00219408_m1 Robo4 was used. Expression was normalized to the endogenous reference TATA-Box binding protein (TBP) (forward 5'-CACGAACCAC GGCACTGATT-3'; reverse 5'-TTTTCTGCTGCCAG TCTGGAC-3'; probe 5'-FAM- TCTTCACTCTTGGC TCCTGTGCACA-TAMRA-3) and HPRT1 (forward primer, 5'-GCTTTCCTTGGTCAGGCAGTA-3'; reverse primer; 5'-GTCTGGCTTATATCCAACACTT CGT-3'; probe, 5'-FAM-GTCTGGCTTATATCCAA CACTTCGT-TAMRA-3'). All TaqMan probes were labeled with 6-Fam reporter dye and Tamra quencher dye. TaqMan gene expression assays were performed as previously described by our group (17).

\section{Western Blot Analysis}

Cells $\left(0.5-1.0 \times 10^{6}\right)$ were directly lysed in a well of a 6 well plate using $100 \mu \mathrm{l} 2 x$ laemmli buffer. The cell lysate was transferred into a $1.5 \mathrm{ml}$ micro tube, sonicated (Branson Sonifier 250), 5\% 2-mercaptoethanol was added and then heated at $95^{\circ} \mathrm{C}$ for 5 minutes. Western Blot was performed as previously described (17). Membranes were incubated at $4^{\circ} \mathrm{C}$ overnight with the antibodies Robo 4 (AF2366, R\&D systems, dilution 1:250) and GAPDH (clone 6C5, MAB374, Merck Millipore, dilution 1:50.000). Afterwards the membrane was incubated with infrared fluorescent dye labeled secondary antibodies (LiCor Biosciences) for 1 hour at room temperature and scanned using the Odyssey infrared imaging system. Densitometric analysis was performed using Odyssey application software (LiCor Biosciences).

\section{[ $\left.{ }^{3} \mathrm{H}\right]$ Thymidine incorporation assay}

Cells were seeded in quintuplicates onto 96-well plates. On the next day, cells were transfected with overexpression and control plasmid as described above for $96 \mathrm{~h}$. $1 \mu \mathrm{Ci} /$ well of $\left[{ }^{3} \mathrm{H}\right]$ thymidine was added to cells overnight. The day thereafter DNA was harvested on 96-well filter plates (UniFilter; Perkin-Elmer), Scintillation fluid $(50 \mu \mathrm{L})$ was added and radioactivity was quantified using Chameleon 5025 liquid scintillation counter (HVD Life Sciences).

\section{Viability assay}

Viability was assessed using WST reagent (Roche) according to the manufacturer's instructions.

\section{Flow cytometry}

Cells were seeded in 6-well plates and transfected with overexpression plasmids or siRNA as described above for $96 \mathrm{~h}$. Afterwards cells were trypsinized and cell pellets were re-suspended in propidium iodide (PI) buffer $(0.2 \%$ Triton-X-100, 2 $\mathrm{ng} / \mathrm{mL}$ Na-Citrate, and $0.1 \mathrm{mg} / \mathrm{mL} \mathrm{PI}$ ) and kept light-protected at $4^{\circ} \mathrm{C}$ for $1 \mathrm{~h}$. Apoptosis was analyzed measuring subG1 peak using FACS Calibur (Becton Dickinson).

\section{Statistical evaluation}

Baseline characteristics as well as histopathological parameters were analyzed descriptively (absolute and relative frequency for qualitative data and mean and SEM for quantitative data). Fisher's exact test was performed for group comparisons. Kaplan Meier product-limit estimation curves for time to recurrence of PCa was produced and groups were compared with the log-rank test. $75 \%$ quartile was used for determination of "high" Robo 4 or Slit 2 expression. Further the online 
BioProfiling Gene Expression Data Mining database $(20,21)$ (GEOSET database ID TCGA_PRAD) for external validation of Robo 4 and Slit 2 prognostic value was used. A significance level of $a=0.05$ (two-tailed) was applied. Statistical analyses were conducted in SPSS, version 22.0 (IBM Corp, Armonk, NY) as well as using Graph Pad Prism Version 5.0.

\section{Results}

\section{Robo 4 and Slit 2 expression in prostate patient tissue:}

Using a TMA containing prostate tumor and paired benign tissue samples of 95 PCa patients who underwent RPE we first investigated potential differences of Robo 4 expression in cancerous compared to benign prostate tissue. Patient characteristics are shown in Table 1, tumor histologies including Gleason Score (GS) and pT stage of biopsy and corresponding RPE specimens are shown in Table 2. Interestingly, we found that Robo 4 expression was significantly increased in prostate tumors of younger patients ( $\leq 60$ years) compared to elderly ( $>60$ years) $(p=0.04)$ while a correlation between pre-surgery PSA values and Robo 4 or Slit 2 expression was not found.

Robo 4 was significantly increased in cancer compared to benign prostate tissue (Figure 1A and Supplementary Figure 1). In addition, we investigated the Robo 4 ligand Slit 2 and also found a significant increased Slit 2 expression in the cancer area of patients compared to benign tissue (Figure 1B and Supplementary Figure 1). The endothelial cell marker CD31 was used as a positive control for blood vessel quantification showing that CD31 levels are higher in tumor tissue compared to non-cancerous prostate tissue $(p=0.0001)$ (Figure $1 C$ and Supplementary Figure 2).

Table 1: Patient characteristics of the TMA

\begin{tabular}{llll}
\hline & mean & median & range \\
\hline Age & 62.9 & 63 & $45-78$ \\
PSA & 6.9 & 5.75 & $1.5-40.6$ \\
fPSA $(\%)$ & 13.2 & 12.1 & $6-39.1$ \\
Prostate volume $(\mathrm{g})$ & 44.6 & 40 & $15-130$ \\
\hline
\end{tabular}

Table 2: Tumor histologies

\begin{tabular}{lcl}
\hline \multicolumn{1}{c}{$\mathbf{n}$} & $\%$ \\
\hline Gleason Score Prostate Biopsy & \\
GS6 & 51 & 53.68 \\
GS7 & 29 & 30.53 \\
GS $\geq 8$ & 8 & 8.42 \\
unkown & 7 & 7.37 \\
Gleason Score Radical Prostatectomy & \\
GS6 & 24 & 25.26 \\
GS7 & 55 & 57.89 \\
GS $\geq 8$ & 16 & 16.85 \\
unkown & - & \\
pT Stage Radical Prostatectomy & \\
pT2a/b & 13 & 13.68 \\
pT2c & 49 & 51.58 \\
pT3 & 32 & 33.68 \\
pT4 & 1 & 1.06 \\
\hline
\end{tabular}

A

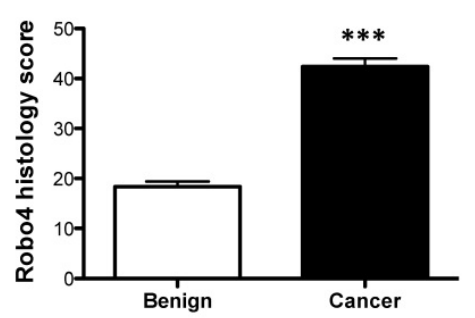

D

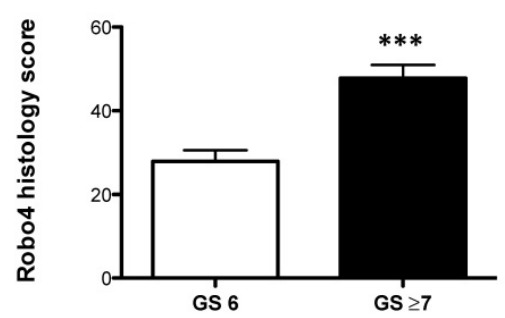

B

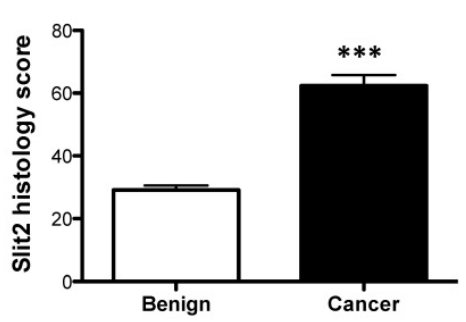

E

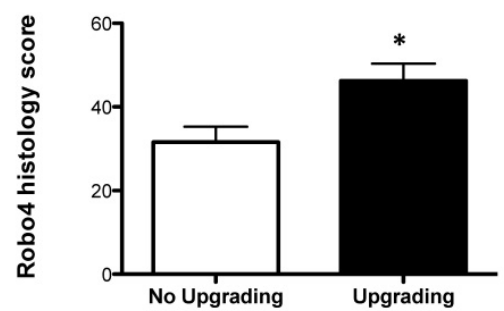

C

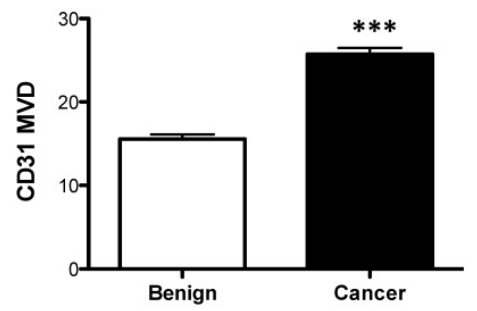

$\mathbf{F}$

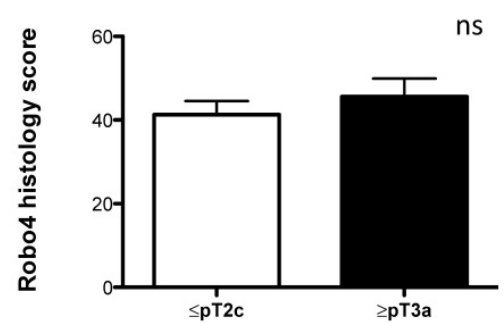

Figure 1: Immunohistochemical analyses of A) Robo 4-, B) Slit 2-staining intensity scores as well as C) CD31 microvessel density (MVD) of radical prostatectomy specimens analyzed according to benign vs. cancer tissue. Robo 4 histology score comparing D) Gleason score (GS), E) GS upgrading and F) pathological stage in the radical prostatectomy specimens. ${ }^{*} \mathrm{p}<0.05 ;{ }^{*} \mathrm{p}<0.01 ;{ }^{* *} \mathrm{p}<0.001 ; \mathrm{n}=95$. 
A

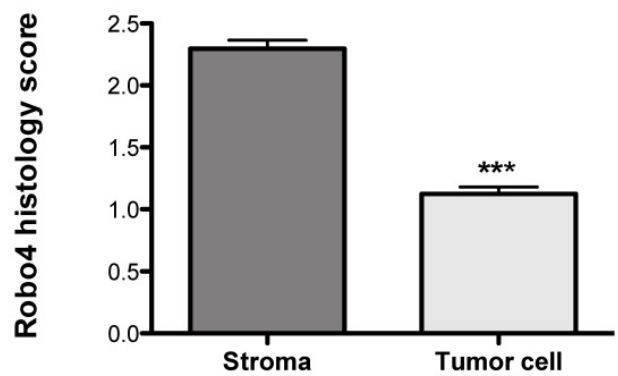

Cancer core
B

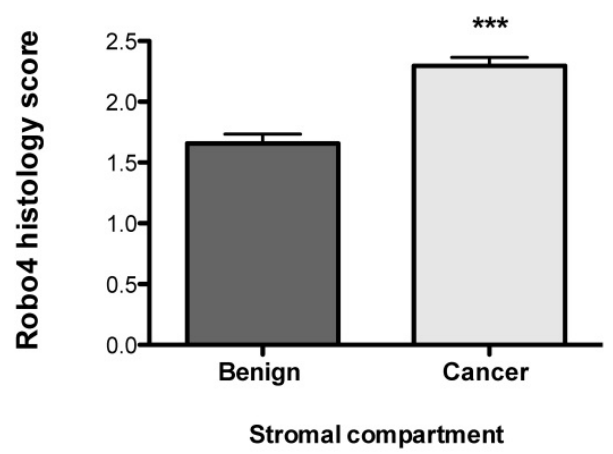

Figure 2: Robo 4 staining intensity scores of a tissue microarray of radical prostatectomy specimens of prostate cancer patients stratified according to cell compartments: A) stroma and tumor cell in cancer cores; B) Robo 4 expression in the stromal compartment of benign and cancer cores; $* * *$ p $<0.001$; $n=95$.

\section{Correlation of Robo 4 and Slit 2 expression with PCa aggressiveness:}

Next we analyzed the impact of Robo 4 on PCa aggressiveness. Thereby our data clearly reveal that high Robo 4 expression is associated with higher GS and thus more aggressive PCa (GS 6 vs. $\geq$ GS 7 : $p=0.0007$ ) (Figure 1D). Moreover, we compared those patients who had a GS upgrade in the RPE specimen from the initial prostate biopsy to those without GS upgrade - again increased Robo 4 expression was predictive for PCa higher aggressiveness expressed by GS upgrade in the RPE specimens $(p=0.04)$ (Figure 1E). However, we did not find differences in Robo 4 expression among patients with organ confined vs. non-organ confined PCa $(\mathrm{p}=0.43)$ (Figure $1 \mathrm{~F})$. In contrast to Robo 4, Slit 2 had no significant correlation concerning PCa aggressiveness, histology or upgrading (data not shown).

\section{Pattern of Robo $\mathbf{4}$ in prostate patient tissue:}

In addition to the total Robo 4 expression on tissue specimens we performed a sub-analysis concerning Robo 4 localization pattern in patients' tissue. Thereby we found that Robo 4 was expressed not only in the tumor stroma but also on cancer cells, however significantly lower $(\mathrm{p}<0.0001)$ (Figure 2A). Moreover, we assessed Robo 4 expression in the stromal compartment of benign and cancerous prostate tissues and found as expected a significant higher expression of Robo 4 in tumor compared to benign stroma $(\mathrm{p}<0.0001)$ (Figure 2B).

To further confirm this TMA based new finding concerning Robo 4 localization also in cancer cells we performed Robo 4 IHC staining also on whole sections of paraffin embedded tissue of $10 \mathrm{RPE}$ specimens (5 low risk PCa, 5 high risk PCa). Thereby we were able to confirm on the above-described compartmentalization of Robo 4 in PCa (Figure 3).

\section{Influence of Robo 4 on tumor recurrence and overall survival after RPE surgery:}

Evaluating the impact of Robo 4 on tumor relapse after RPE, we compared total Robo 4 tissue expression in patients with $(\mathrm{n}=16)$ and without $(\mathrm{n}=79)$ biochemical recurrence (BCR) after surgery (postoperative PSA increase $>0.2 \mathrm{ng} / \mathrm{ml}$ ). Thereby our data reveal no significant differences among both groups as patients with BCR harbored a mean Robo 4 staining score of 38.3, while those patients without a BCR after RPE had a mean Robo 4 staining score of 43.7 (ns) (Figure 4A). In line with Robo 4, also Slit 2 expression was comparable in patients without BCR compared to those with BCR (53.8 pg/ml vs 67.1) (ns) (Figure 4B).

Next we stratified those patients with a BCR $(n=16)$ according to low and high Robo 4 expression ( $75 \%$ quartile). Although the finding of this analysis is underpowered and no statistical significance could be achieved, we observed a trend that patients with high Robo 4 expression experience a longer time period to BCR than those with low Robo 4 expression (Figure 4C) suggesting that Robo 4 might have a role in vascular stabilization leading to reduced tumor recurrence as reported for other tumor entities (22). The same trend was also noted for the Robo 4 ligand Slit 2 (Figure 4D).

As in our analyzed patient collective, no patients died from PCa during the study period, we assessed the impact of mRNA Robo 4 and Slit 2 expression on overall survival (OS) using the online BioProfiling Gene Expression Data Mining database $(18,19)$ (GEOSET database ID TCGA_PRAD) including 147 patients (clinical variable white race). Briefly we found that neither Robo 4 nor Slit 2 expression had a significant impact on OS in PCa patients (Figure 5). 


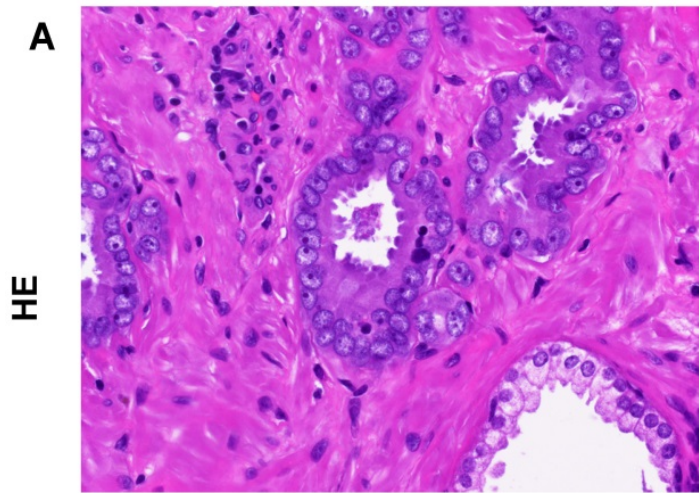

B
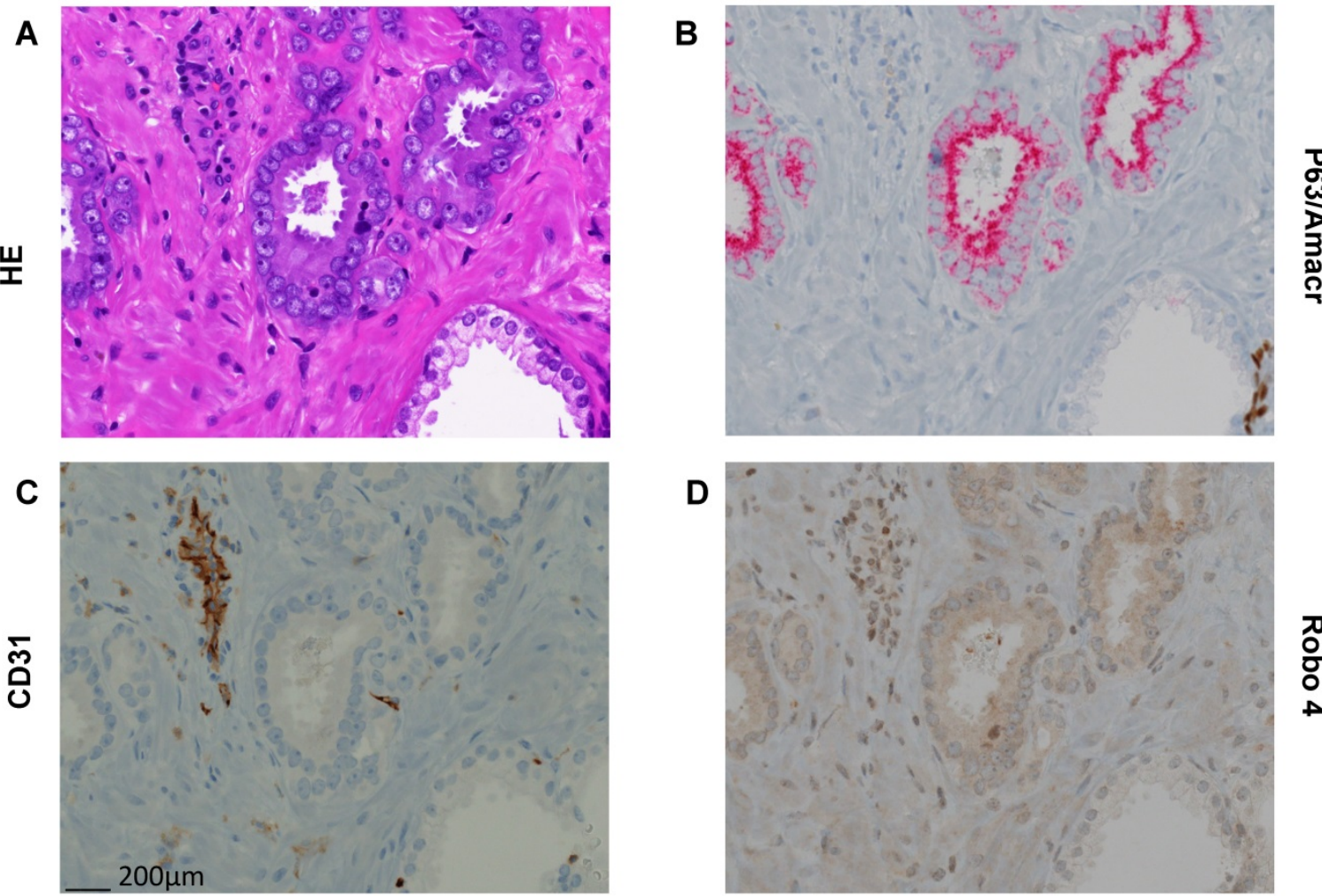

D

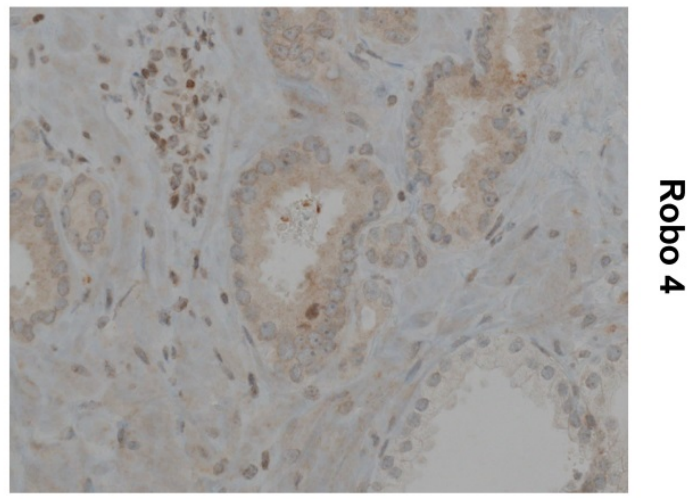

Figure 3: Representative pictures of Hematoxilin/Eosin (HE) and immunhistochemical stainings (AMACR/p63 doublestaining, CD31, Robo 4 on paraffin embedded tissue of a radical prostatectomy specimen. A) HE staining with clearly different morphology in benign and cancerous glands. B) P63/AMACR doublestaining demonstrating benign glands as AMACR negative with p63 positive basal cells (dark brown), while cancer glands are AMACR positive (red) and p63 negative. C) Endothelial cells with typical CD31 positivity. D) Robo 4 expression is missing/weak in benign and intermediate/strong in cancer. In addition, endothelial and stromal cells show Robo 4 positivity (in cancer glands surrounding stroma stronger than in benign glands surrounding stroma). Scale bar $=200 \mu \mathrm{m}$.

A

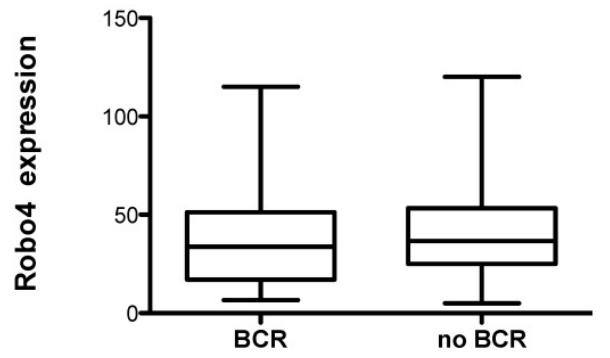

C

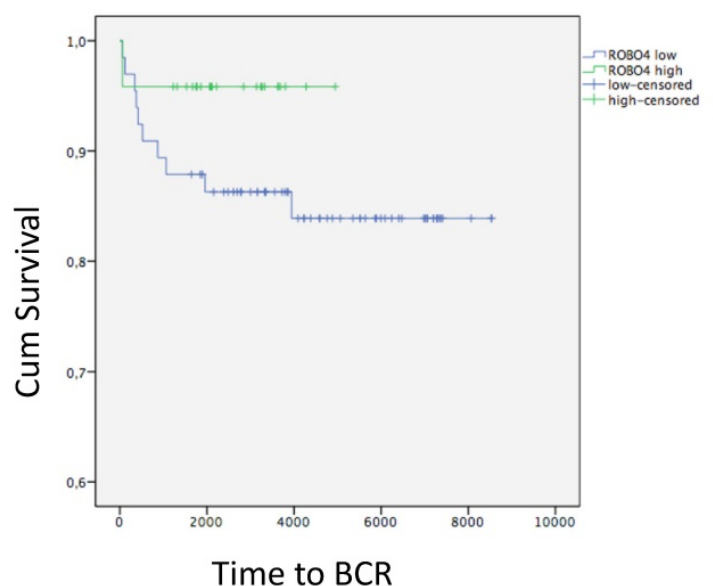

B

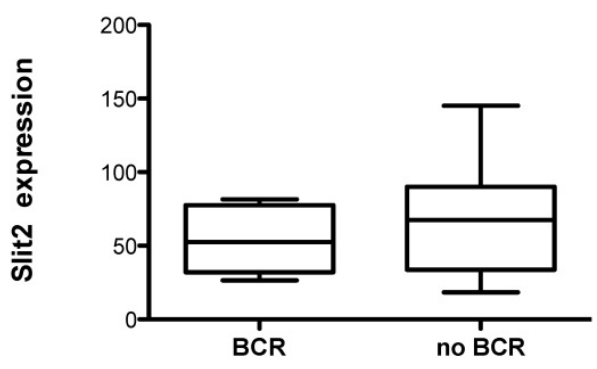

D

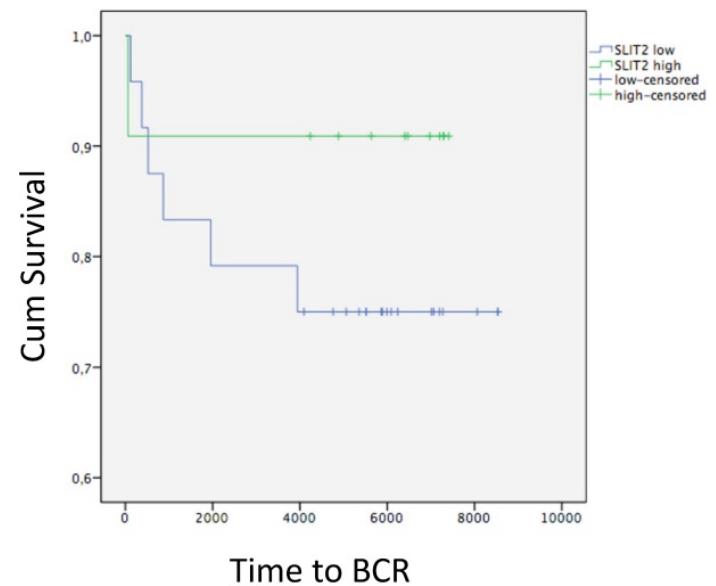

Figure 4: Box plots showing A) Robo 4 and B) Slit 2 expression in patients with a biochemical recurrence (BCR) after radical prostatectomy. Data represent mean + SEM. Kaplan Meier curves of high (green) versus low (blue) C) Robo 4 and D) Slit 2 expression. Differences among both groups were applied by log-rank test. 75\% quartile was used for determination of "high" Robo 4 or Slit 2 expression; $n=16$. 
A

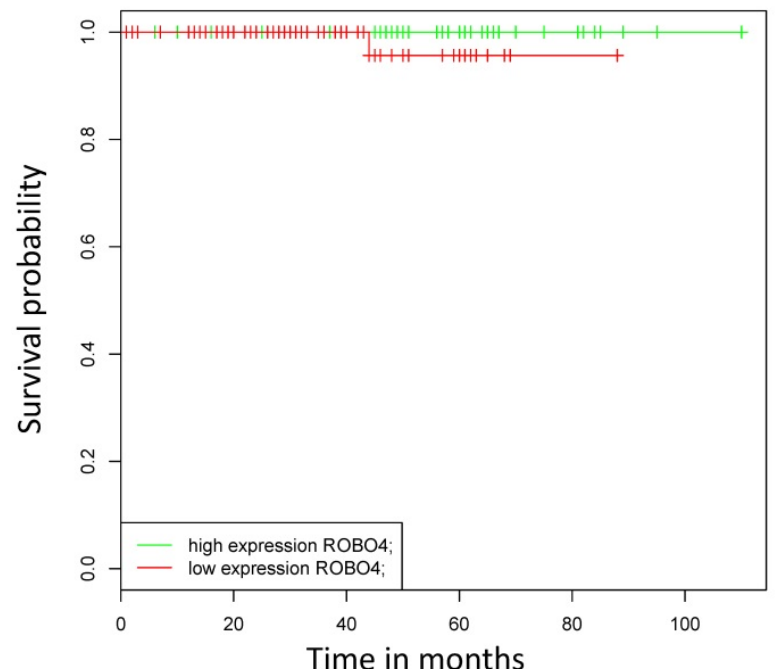

B

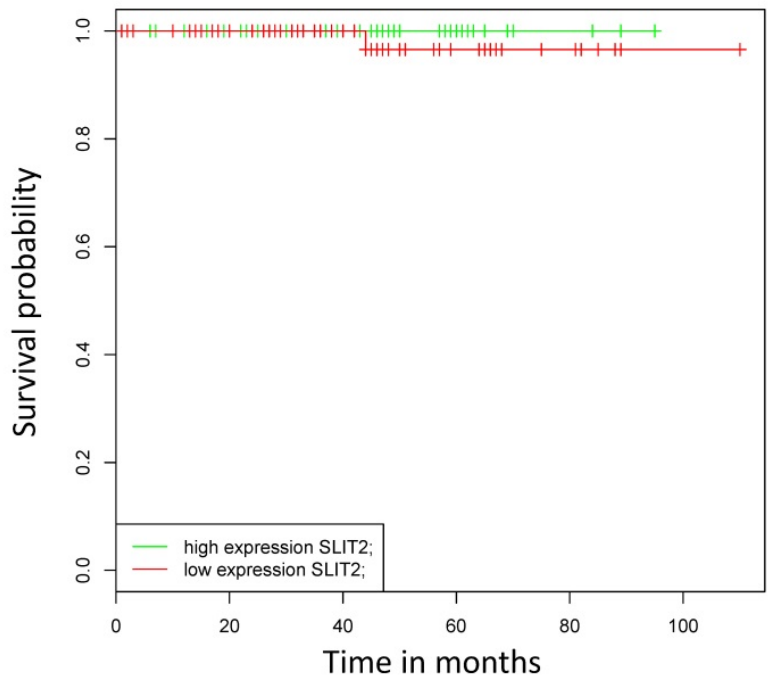

Figure 5: Kaplan Meier curves analyzing overall survival of patients with high (green) versus low (blue) A) Robo 4 and B) Slit 2 expression validated in an external dataset (TCGA_PRAD, race white); $n=147$.

A

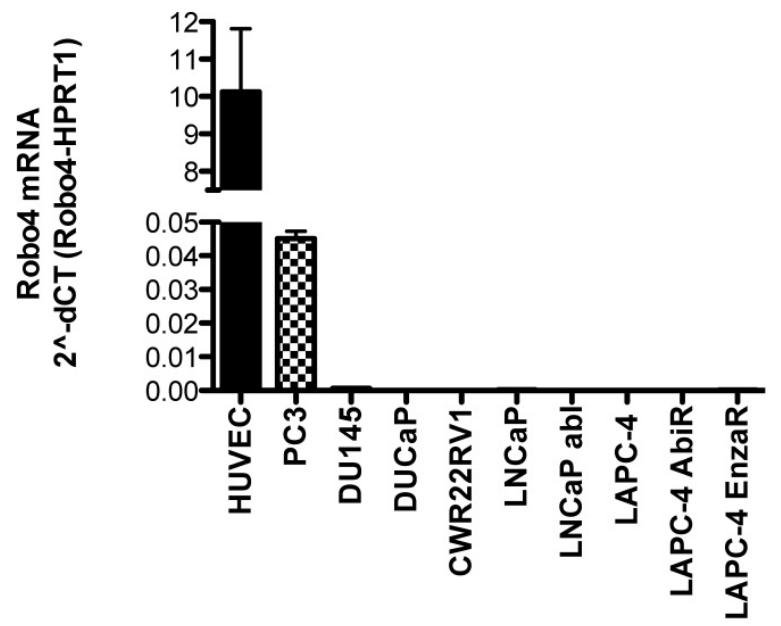

B

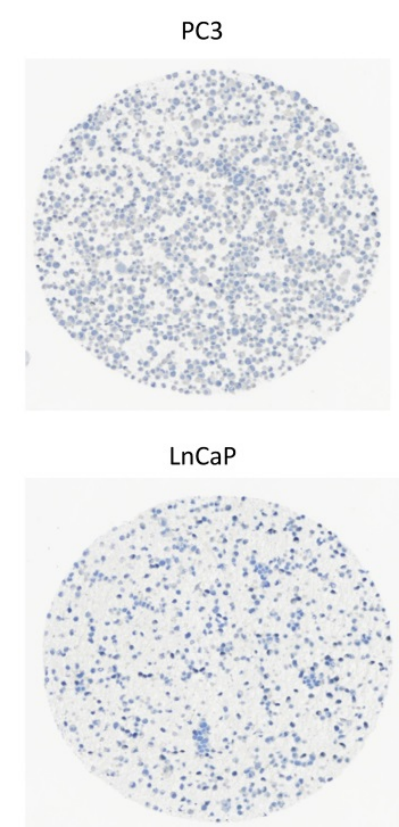

Figure 6: A) Robo 4 mRNA expression in different prostate cancer cell lines as well as in HUVEC used as control cell line, $n=3$; B) Robo4 protein expression on fixed embedded PCa PC3 and LNCaP cells.

\section{Robo 4 expression in PCa cell lines:}

Based on the IHC findings that Robo 4 is expressed also on PCa tumor cells, we tested the endogenous mRNA expression of Robo 4 in different PCa cell lines derived from metastatic PCa. As control we used human umbilical endothelial cells (HUVEC) previously described to express Robo 4 (Figure 6) (9, 22).

qRT-PCR analysis revealed that the aggressive PCa cell line PC3 (derived from bone metastasis of a PCa patient) expresses Robo 4 on both mRNA level (Figure 6A) as well as on protein level (Figure $6 \mathrm{~B}$ ). Also Robo 4 immunofluorescence on PC3 cells available in the human protein atlas confirmed a Robo 4 positivity on PC3 cells (data from the HPA065212 AK/Atlas (www.proteinatlas.org/ENSG00000154133 -ROBO4/cell\#img).

\section{Functional impact of Robo 4 overexpression in PCa cell lines:}

As we observed that Robo 4 is expressed also on tumor cells rather than exclusively on endothelial cells, we aimed to evaluate the functional role of Robo 4 on PCa. For this reason, a transient Robo 4 overexpression and downregulation system was established (Supplementary Figure 3 B-D) and the 

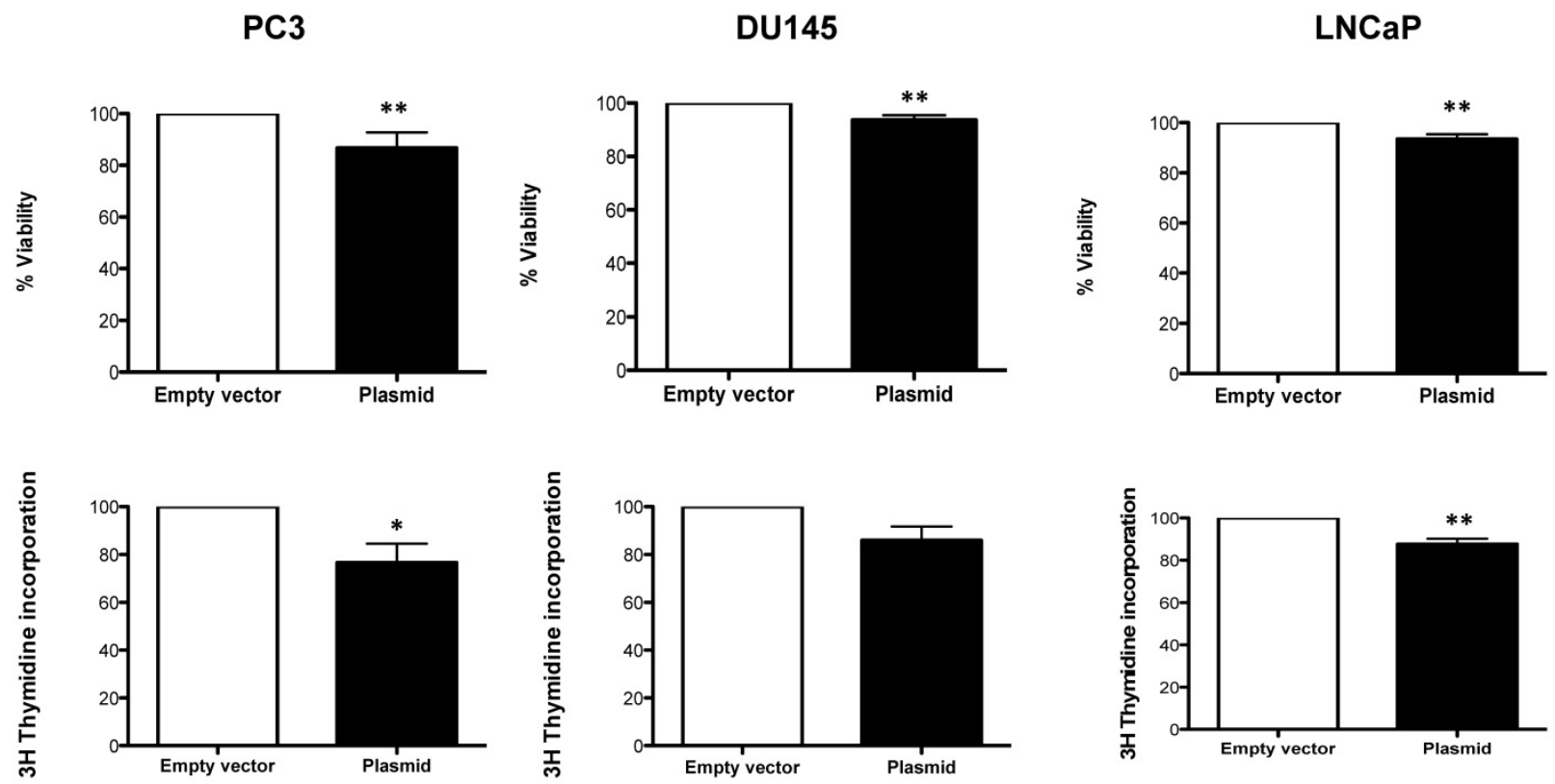

Figure 7: A) \% Viability and B) \% Cell proliferation upon overexpression of Robo 4 in PC3, DU145 and LNCaP cells; data from $\geq 3$ independent experiments, $*_{p}<0.05 ; * * p<0.01 ; * * * p<0.001$.

functional impact of Robo 4 overexpression in three different PCa cell lines was analyzed.

We found, that overexpression of Robo 4 leads to a significant decrease in cell viability (Figure 7A-C). Also cell proliferation was significantly decreased (in 2 of 3 measured PCa cell lines) upon Robo 4 overexpression (Figure 7D-F).

Moreover, we addressed the question if $\mathrm{AR}$ positive and AR negative cell lines act differently when Robo 4 is overexpressed. However, we did not find any significant differences $(p=0.1)$ among $A R$ positive (LNCaP) and AR negative cell lines (DU145 and PC3) speculating that the AR status is not influencing the effects of Robo 4 itself (data not shown).

Furthermore, we addressed the impact of Robo 4 overexpression and downregulation on apoptosis in PC3 cells. Thereby, we did not observe any significant changes and concluded that modulating Robo 4 protein expression has no impact on apoptosis in the investigated cell model (Supplementary Figure 4).

\section{Discussion}

The present study investigates for the first time the impact of Robo 4 and its ligand Slit 2 in PCa aggressiveness both in vitro as well as in the clinical setting. Interestingly, we found that the Robo 4 / Slit 2 axis has an exceptional role in PCa biology exerting additional functions to the well-described role of Robo 4 / Slit 2 signaling in tumor angiogenesis (4, 22-25).

We show, that in contrast to other tumor entities, that did not find Robo 4 expression in tumor cells that the PC3 PCa cancer cell line expresses Robo 4, whenever at a significant lower level compared to HUVEC (other tested PCa cell lines were negative, Figure 6) (24).

Generally, in endothelial cell biology the importance of Robo 4 is well characterized revealing that Robo 4 acts as a receptor for Slit 2 thereby modulating VEGFR2 signaling and inhibiting vascular permeability $(23,24,27,28)$. Recently, it has been shown in breast cancer, that endothelial Robo 4 suppresses tumor angiogenesis and protects vascular integrity. In addition, using an in vivo animal model, Robo 4 knockout led to increased tumor angiogenesis proving that Robo 4 is a main regulator of tumor angiogenesis (29). Moreover, quantification of Robo 4 expression levels in primary tumor samples showed higher Robo 4 expression in malignant tissue compared to normal adjacent tissue in several cancer entities. For example Cai et al. demonstrated, that Robo 4 was significantly upregulated in glioma tissues compared with normal brain tissue (28). Further total Robo 4 expression was significantly higher in bone marrow specimens of acute myeloid leukemia patients compared to normal bone marrow donors (30). These findings are in line with our observations in PCa tissue, where Robo 4 is higher expressed in cancerous tissue compared to normal prostate tissue, respectively. Furthermore, we observed that higher GS (reflecting more aggressive tumors) correlates with higher Robo 4 expression. That's an intriguing finding, as we observed in vitro that Robo 4 overexpressing PC3 cancer cells show a significant reduction in cell proliferation and viability. 
Earlier reports have documented especially for Slit 2 to be a tumor suppressor gene often lost in tumor progression; however the role of Robo 4 in this scenario is still conflicting $(31,32)$. In some cancer entities including non-small cell lung cancer, high Robo 4 expression (no analyses concerning compartmentalization) was associated with good prognosis, mainly attributed to vascular normalization and reduction of metastasis formation (22).

Although not significant, we observed in a small patient cohort, that PCa patients with low Robo 4 and Slit 2 expression tend to have increased BCR rates compared to those patients with increased Robo 4 and Slit 2 levels. In general, PCa has a favorable disease course and up to $84 \%$ and $74 \%$ of patients have a 5year and 10-year disease free survival rate, respectively (33). In line with these findings, also in the present study only 16/95 patients developed a $\mathrm{BCR}$, thus limiting the explanatory power and statistical significance of this interesting finding. Nevertheless, we can show for the first time a potential protecting impact of Robo 4 concerning tumor recurrence. We are completely aware that the present data are only hypothesis generating and have to be validated in a larger prospective study.

As first step for external validation of our own data, we used the online BioProfiling Gene Expression Data Mining database $(20,21)$ where be observed even a trend towards a protecting role of Robo 4 concerning recurrence (OS was analyzed). In contrast to these findings, there is evidence that higher Robo 4 expression might be a marker for poor prognosis e.g. in acute myeloic leukemia high expression of Robo 4 was associated with a significantly shorter OS as that of patients with Robo 4 low expression (30). However, one has to consider that- in line with all previous studies on Robo 4- bulk tissue samples were used and therefore no specific conclusion concerning cell subtype specific Robo 4 expression can be drawn. Future approaches as single cell analyses may shed light on compartment specific Robo 4 expression in various cancers.

In general, the role of Robo 4 seems to be pleiotropic and highly dependent on the tumor microenvironment. In line with this hypothesis we are able to show that Robo 4 is weakly expressed in prostate benign cell types highlighting the context dependency. Next, TMA analysis proved that Robo 4 staining is located in the tumor microenvironment however more diffuse as CD31 MVD quantification, reflecting the prostate vasculature alone. Slit 2 expression was more prominent in the glands themselves thereby leading to the hypothesis that Slit 2 arises from the cancer cells and acts context dependent on different cell types of the tumor microenvironment as well as in an autocrine manner on the cancer cells (negative feedback).

Briefly, in the present study we observed a dual effect of Robo 4 in PCa as I) it is associated with more aggressive cancers in the localized disease setting as well as a hypothesis generating trend towards II) a protective effect of Robo 4 concerning tumor recurrence and OS after RPE. Further studies in larger patient collectives are warranted to proof this hypothesis.

\section{Conclusion}

In summary we observed that Robo 4 plays a considerable role in PCa development as it is expressed in cancer epithelial cells as well as in the surrounding tumor stroma. Moreover, higher histological tumor grade was associated with increased Robo 4 expression; controversially patients with high Robo 4 tend to exert lower biochemical recurrence possibly reflecting a protective role of Robo 4.

\section{Supplementary Material}

Supplementary figures.

http://www.medsci.org/v16p0115s1.pdf

\section{Acknowledgements}

We thank Irma Sottas for help with TMA construction.

\section{Ethics approval and consent to participate}

Local Ethics committee Medical University Innsbruck, Austria (UN3174, AM3174).

\section{Funding}

MUI Start, Medical University Innsbruck, Austria- grant to I. Heidegger.

\section{Authors' contributions}

Andreas Pircher: General idea, funding, data interpretation, TMA analyses, planning experiments, manuscript writing

Georg Schäfer: Help with TMA generation, data interpretation, manuscript writing

Andrea Eigentler: Generation of cell culture data

Renate Pichler: Collection of follow up patient data

Martin Puhr: Help with TMA acquisition, manuscript writing

Eberhard Steiner: Help with statistical analyses

Eberhard Gunsilius: Providing antibodies, supervision

Wolfgang Horninger: Supervision

Helmut Klocker: Supervision, planning 
experiments, manuscript writing

Isabel Heidegger: Funding, data interpretation, planning experiments, manuscript writing

\section{Competing Interests}

The authors have declared that no competing interest exists.

\section{References}

1. Wang D, Tindall DJ. Androgen action during prostate carcinogenesis. Methods Mol Biol. 2011;776:25-44.

2. de Brot S, Ntekim A, Cardenas R, James V, Allegrucci C, Heery DM, et al. Regulation of vascular endothelial growth factor in prostate cancer. Endocr Relat Cancer. 2015;22(3):R107-23.

3. Schweizer MT, Carducci MA. From bevacizumab to tasquinimod: angiogenesis as a therapeutic target in prostate cancer. Cancer J. 2013;19(1):99-106.

4. Pircher A, Johrer K, Kocher F, Steiner N, Graziadei I, Heidegger I, et al. Biomarkers of evasive resistance predict disease progression in cancer patients treated with antiangiogenic therapies. Oncotarget. 2016;7(15):20109-23.

5. Pircher A, Hilbe W, Heidegger I, Drevs J, Tichelli A, Medinger M. Biomarkers in tumor angiogenesis and anti-angiogenic therapy. Int $\mathrm{J}$ Mol Sci. 2011;12(10):7077-99.

6. Jayson GC, Kerbel R, Ellis LM, Harris AL. Antiangiogenic therapy in oncology: current status and future directions. Lancet. 2016;388(10043):518-29.

7. Heidegger I, Pircher A, Bektic J. Is there a role for angiogenesis inhibition in prostate cancer? memo - Magazine of European Medical Oncology. 2014;7(4):214-8.

8. St Croix B, Rago C, Velculescu V, Traverso G, Romans KE, Montgomery E, et al. Genes expressed in human tumor endothelium. Science. 2000;289(5482):1197-202.

9. Seth P, Lin Y, Hanai J, Shivalingappa V, Duyao MP, Sukhatme VP. Magic roundabout, a tumor endothelial marker: expression and signaling. Biochem Biophys Res Commun. 2005;332(2):533-41.

10. Pircher A, Wellbrock J, Fiedler W, Heidegger I, Gunsilius E, Hilbe W. New antiangiogenic strategies beyond inhibition of vascular endothelial growth factor with special focus on axon guidance molecules. Oncology. 2014;86(1):46-52.

11. Mehlen P, Delloye-Bourgeois C, Chedotal A. Novel roles for Slits and netrins: axon guidance cues as anticancer targets? Nat Rev Cancer. 2011;11(3):188-97.

12. Miyata Y, Mitsunari K, Asai A, Takehara K, Mochizuki Y, Sakai H. Pathological significance and prognostic role of microvessel density, evaluated using CD31, CD34, and CD105 in prostate cancer patients after radical prostatectomy with neoadjuvant therapy. Prostate. 2015;75(1):84-91.

13. Culig Z, Hoffmann J, Erdel M, Eder IE, Hobisch A, Hittmair A, et al. Switch from antagonist to agonist of the androgen receptor bicalutamide is associated with prostate tumour progression in a new model system. Br J Cancer. 1999;81(2):242-51.

14. Hoefer J, Akbor M, Handle F, Ofer P, Puhr M, Parson W, et al. Critical role of androgen receptor level in prostate cancer cell resistance to new generation antiandrogen enzalutamide. Oncotarget. 2016.

15. Puhr M, Hoefer J, Eigentler A, Ploner C, Handle F, Schaefer G, et al. The glucocorticoid receptor is a key player for prostate cancer cell survival and a target for improved anti-androgen therapy. Clin Cancer Res. 2017.

16. Puhr M, Hoefer J, Schafer G, Erb HH, Oh SJ, Klocker $\mathrm{H}$, et al. Epithelial-to-mesenchymal transition leads to docetaxel resistance in prostate cancer and is mediated by reduced expression of miR-200c and miR-205. Am J Pathol. 2012;181(6):2188-201.

17. Heidegger I, Ofer P, Doppler W, Rotter V, Klocker H, Massoner P. Diverse functions of IGF/insulin signaling in malignant and noncancerous prostate cells: proliferation in cancer cells and differentiation in noncancerous cells. Endocrinology. 2012;153(10):4633-43.

18. Kern J, Bauer M, Rychli K, Wojta J, Ritsch A, Gastl G, et al. Alternative splicing of vasohibin-1 generates an inhibitor of endothelial cell proliferation, migration, and capillary tube formation. Arterioscler Thromb Vasc Biol. 2008;28(3):478-84

19. Schopf B, Schafer G, Weber A, Talasz H, Eder IE, Klocker H, et al. Oxidative phosphorylation and mitochondrial function differ between human prostate tissue and cultured cells. FEBS J. 2016;283(11):2181-96.

20. Antonov AV. BioProfiling.de: analytical web portal for high-throughput cell biology. Nucleic Acids Res. 2011;39(Web Server issue):W323-7.

21. Amelio I, Knight RA, Lisitsa A, Melino G, Antonov AV. p53MutaGene: an online tool to estimate the effect of p53 mutational status on gene regulation in cancer. Cell Death Dis. 2016;7:e2148.

22. Pircher A, Fiegl M, Untergasser G, Heidegger I, Medinger M, Kern J, et al. Favorable prognosis of operable non-small cell lung cancer (NSCLC) patients harboring an increased expression of tumor endothelial markers (TEMs). Lung Cancer. 2013;81(2):252-8
23. Jones CA, London NR, Chen H, Park KW, Sauvaget D, Stockton RA, et al. Robo4 stabilizes the vascular network by inhibiting pathologic angiogenesis and endothelial hyperpermeability. Nat Med. 2008;14(4):448-53.

24. Yadav SS, Narayan G. Role of ROBO4 signalling in developmental and pathological angiogenesis. Biomed Res Int. 2014;2014:683025.

25. Borrell V, Cardenas A, Ciceri G, Galceran J, Flames N, Pla R, et al. Slit/Robo signaling modulates the proliferation of central nervous system progenitors. Neuron. 2012;76(2):338-52.

26. Grone J, Doebler O, Loddenkemper C, Hotz B, Buhr HJ, Bhargava S. Robo1/Robo4: differential expression of angiogenic markers in colorectal cancer. Oncol Rep. 2006;15(6):1437-43.

27. Koch AW, Mathivet T, Larrivee B, Tong RK, Kowalski J, Pibouin-Fragner L, et al. Robo4 maintains vessel integrity and inhibits angiogenesis by interacting with UNC5B. Dev Cell. 2011;20(1):33-46.

28. Cai H, Liu W, Xue Y, Shang X, Liu J, Li Z, et al. Roundabout 4 regulates blood-tumor barrier permeability through the modulation of ZO-1, Occludin, and Claudin-5 expression. J Neuropathol Exp Neurol. 2015;74(1):25-37.

29. Zhao H, Ahirwar DK, Oghumu S, Wilkie T, Powell CA, Nasser MW, et al. Endothelial Robo4 suppresses breast cancer growth and metastasis through regulation of tumor angiogenesis. Mol Oncol. 2016;10(2):272-81.

30. Chen YK, Hou HA, Tang JL, Jhuang JY, Lai YJ, Lee MC, et al. Clinical and prognostic implications of Roundabout 4 (robo4) in adult patients with acute myeloid leukemia. PLoS One. 2015;10(3):e0119831.

31. Yu J, Zhang X, Kuzontkoski PM, Jiang S, Zhu W, Li DY, et al. Slit2N and Robo4 regulate lymphangiogenesis through the VEGF-C/VEGFR-3 pathway. Cell Commun Signal. 2014;12:25.

32. Dallol A, Krex D, Hesson L, Eng C, Maher ER, Latif F. Frequent epigenetic inactivation of the SLIT2 gene in gliomas. Oncogene. 2003;22(29):4611-6.

33. Han M, Partin AW, Pound CR, Epstein JI, Walsh PC. Long-term biochemical disease-free and cancer-specific survival following anatomic radical retropubic prostatectomy. The 15 -year Johns Hopkins experience. Urol Clin North Am. 2001;28(3):555-65. 